\title{
A Special-purpose Computer for the Monte Carlo Simulation using Random Numbers with an Arbitrary Distribution
}

\author{
Member Takeo Ishikawa (Gunma University) \\ Non-member Yoshio Yamaguchi (Gunma University) \\ Non-member Takashi Yabe (Gunma University)
}

Key words : Special-purpose computer, Monte Carlo method, Random number

\section{Introduction}

The Monte Carlo method is now the powerful and commonly used technique for analyzing statistical problems in many fields ${ }^{(1)}$. However, when high accuracy is required, the cost of performing the computation on a general-purpose computer including a supercomputer is prohibitive, because the accuracy obtained in the Monte Carlo calculation is proportional to $1 / \sqrt{N}$, where $N$ is the number of statistical samples. As an alternative to the general-purpose computer, several special-purpose computers for the Monte Carlo simulation have been constructed ${ }^{(2)}$. However, they handle only the Ising models by using random numbers with the uniform distribution. This paper develops a specialpurpose computer which can handle a large class of the Monte Carlo problems by using random numbers with an arbitrary distribution.

\section{Hardware design}

It is clear that a completely hardwired system that can perform the same thing as a full program for the Monte Carlo simulation cannot be built. However, the part for generating random numbers can be realized by a common hardwired system, because all Monte Carlo simulations need random numbers. As the algorithm for the rest depends on the application which we want to study, it should be organized by software. In the present work, several transputers are utilized, because they can easily perform the parallel calculation. If we want to study a special application, the transputers can be replaced by a hardwired system.

Figure 1 shows the block diagram of the special-purpose computer which we have designed and built. Transputers are connected to a general-purpose host computer and to the random number generator(RNG) developed by the authors $^{(3)}$. The host computer sends transputers a compiled program for the Monte Carlo simulation. The program is written in parallel $\mathrm{C}$ and/or parallel Fortran language avail- able on the transputers. The transputers perform in parallel the Monte Carlo simulation using random numbers with the arbitrary distribution generated by the RNG. The topology of the array of the transputers should be decided according to the application.

The RNG is the system for the high speed generation of random numbers with the arbitrary distribution. It prepares two tables of random numbers and stores them in two memories, and then picks out two random numbers and assembles them, resulting a new random number. One of our originalities in the RNG can be found in the method to make the tables ${ }^{(3)}$. Figure 2 shows the block diagram of the RNG. ROMs $X$ and $Y$ store the tables. Counters \#2 and \#3 count one hundred from the value given by ROMs Hx and $\mathrm{Hy}$ in order to produce random numbers from ROMs $\mathrm{X}$ and $\mathrm{Y}$.

\section{Performance}

We are currently using two transputers $(30 \mathrm{MHz}$ T805) and EPSON personal computer (PC386) as the host computer. As the time of one cycle of the RNG is $200 \mathrm{~ns}$, this system can generate 5 million random numbers a second.

In order to show the basic performance of the developed computer, we calculate the following integration using the importance sampling:

$$
I=\int_{0}^{1} e^{x} d x .
$$

This integration can be estimated as follows:

$$
I=\frac{3}{2 N} \sum_{i=1}^{N} \frac{e^{\eta_{i}}}{\left(1+\eta_{i}\right)}
$$

where $\eta_{i}$ is a random number with the density function of $\frac{2}{3}(1+x)$ in $[0,1]$. Transputer \#1 gets a random number $\eta_{i}$ from the RNG and sends it to transputer \#2. Transputer \#2 calculates the equation (2). These two transputers perform in parallel the calculation. The computational time of the transputer \#1 per random number is $15.0 \mu \mathrm{sec}$, and 


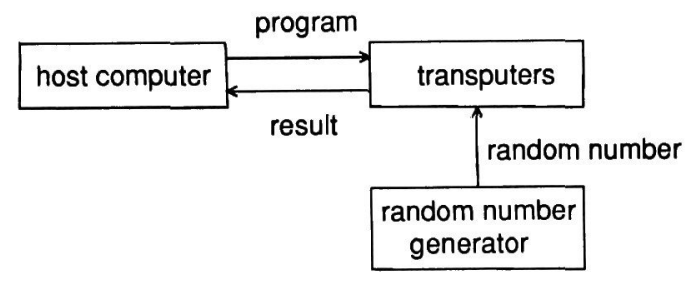

Fig.1 Block diagram of the computer.

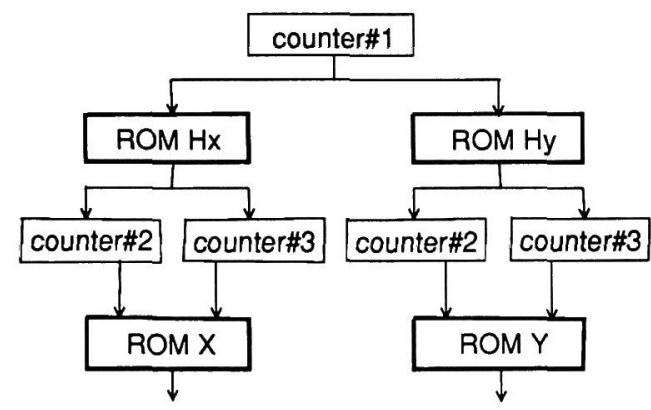

Fig.2 Block diagram of the random number generator.

that of the transputer \#2 is $44.8 \mu$ sec. The time to transfer data between two transputers is $4.6 \mu s e c$. Therefore, the computational time per random number is $49.4 \mu \mathrm{sec}$.

\section{Conclusion}

A special-purpose computer with the RNG and several transputers has been developed to compute the Monte Carlo method. It can handle a large class of the Monte Carlo problems. Some applications will be studied in the future.

\section{Acknowledgment}

Part of this work was supported by the Grant-in-Aid for Encouragement of Young Scientists from the Ministry of Education, Science and Culture.

(Manuscript received June 25, '93)

\section{References}

(1) K.Binder and D.W.Heermann : Monte Carlo simulation in statistical physics, Springer-Verlag (1992).

(2) M.Taiji, N.Ito and M.Suzuki : "Special purpose computer system for Ising models", Rev. Sci. Instrum. 59, 2483 (1988).

(3) T.Ishikawa et al. : "A method for the high-speed generation of random numbers with arbitrary distributions", Comp. Phys. Comm. 70, 501 (1992).

\section{Takeo Ishikawa (Member)}

He was born January 1, 1956. He received the B.E. degree from Saitama University in 1978, and the M.E. and D.E. degrees from Tokyo Institute of Technology in 1980 and 1983, respectively. From 1983 to 1991, he was a Research Associate at Gunma University. He has been an Associate Professor there since 1991. He was a visiting researcher at the University of Toronto from 1990 to 1991. His current research interests are in the area of numerical analysis of electromagnetic field and computer hardware.

$\mathrm{He}$ is a member of the Institute of Electrical and Electronics Engineers and the Japan Society for Simulation Technology.

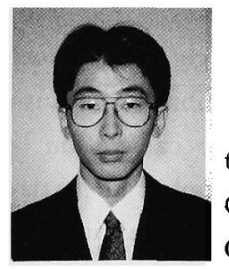

Yoshio Yamaguchi (Non-member)

He was born March 1, 1970. He received the B.E. degree in electronic engineering from Gunma University in 1993, and joined Anritsu Corporation.

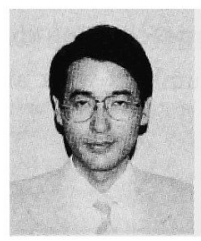

Takashi Yabe (Non-member)

He was born January 10, 1950. He graduated Department of Mechanics \& Physics at Tokyo Institute of Technology in 1973 and obtained Ph.D in 1980 at Tokyo Institute of

Technology.

He engaged in the computer simulation of laser-driven fusion for 10 years at Institute of Laser Engineering, Osaka University. His current research interests are in the area of computational mathematics and computer hardware. He is now a full professor at Department of Electronic Engineering, Gunma University. Doctor of Engineering. 